TRANSACTIONS OF THE

AMERICAN MATHEMATICAL SOCIETY

Volume 147, February 1970

\title{
SEMIGROUPS OF RIGHT QUOTIENTS OF TOPOLOGICAL SEMIGROUPS
}

\author{
BY \\ HANNS JOACHIM WEINERT
}

Introduction. Let $S=(S, \cdot)$ be a semigroup and $T=Q_{r}(S, \Sigma)$ a semigroup of right quotients of $S$ with respect to a subsemigroup $\Sigma$ of $S$ (cf. §2). Suppose that $S$ is equipped with a topology $\subseteq$ which makes $(S, \cdot, \subseteq)$ into a topological semigroup. The purpose of this paper is to investigate topologies $\mathfrak{T}$ on $T$ with the properties that $(T, \cdot, \mathfrak{I})$ is a topological semigroup and that the relative topology $\mathfrak{I} \mid S$ obeys $\mathfrak{I} \mid S=\mathfrak{S}$ or more generally $\mathfrak{T} \mid S \subseteq \mathfrak{S}$.

There is a related question if one assumes that there exists even a group $T=Q_{r}(S, S)$ of right quotients and considers topologies $\mathfrak{I}$ on $T$ which make $(T, \cdot, \mathfrak{T})$ into a topological group. Several authors have dealt with this topic [9], [8], especially in the commutative case [2], [4], [6], [7]. We shall speak later about some of their results, for which we obtain generalizations.

The first part of this paper starts with the following question: Take $(S, \cdot, \subseteq)$ and $T=Q_{r}(S, \Sigma)$ as above. Does there exist a topology $\mathfrak{I}$ on $T$, such that $(T, \cdot, \mathfrak{T})$ is a topological semigroup, $\mathfrak{T} \mid S=\mathfrak{S}$, and $S$ is $\mathfrak{T}$-open ? We obtain (cf. $\S 3$, Theorem 1) that this is the case if, and only if, all left and right translations by elements $\alpha \in \Sigma$ are open mappings in $(S, \cdot, \mathfrak{S})$. Moreover, there is only one topology $\mathfrak{I}$ of this kind, and $\mathfrak{T}$ can be defined by the base

$$
\left\{U \alpha^{-1} \mid U \subseteq S \text { and open, } \alpha \in \Sigma\right\} \text {. }
$$

But, if $G$ is a subgroup of $T$ (for instance, the unique maximal one or $T$ itself if $T$ happens to be a group), $(G, \cdot, \mathfrak{I} \mid G)$ need not be a topological group. In $\S 5$ (cf. Theorem 3) we give necessary and sufficient conditions for the continuity of $g \rightarrow g^{-1}$ in $(G, \cdot, \mathfrak{I} \mid G)$ for an arbitrary subgroup $G$ of $T$. These conditions are generalizations of a condition $F$ introduced by Rothman [7], and we generalize his Main Theorem (given in [7, p. 200], for the commutative case) as follows: Let $T=Q_{r}(S, S)$ be a group of right quotients of a semigroup $(S, \cdot, \mathcal{S})$, then $F$ is necessary and sufficient for the existence of a topology $\mathfrak{I}$ on $T$ which makes $(T, \cdot, \mathfrak{T}$ ) into a topological group such that $\mathfrak{I} \mid S=\subseteq$ and $S$ is $\mathfrak{I}$-open (cf. Theorem 4). Moreover, by our considerations such a topology (obtained in [7] using [8]) is unique and coincides with the topology above for $\Sigma=S$ (cf. also Theorem 12).

In the second part $(\S 6, \S 7)$ we consider the general problem stated at the beginning

Received by the editors July 31, 1968.

Copyright (C) 1970, American Mathematical Society 
of the introduction. From Theorem 5 follows that it is meaningful to look at families of the kind

$$
\left\{U \Omega^{-1} \mid U \subseteq S \text { and } \Xi \text {-open, } \Omega \subseteq \Sigma \text { and } 3 \text {-open }\right\},
$$

where $\mathfrak{g}$ is a topology on $\Sigma$ which makes $(\Sigma, \cdot, \mathfrak{z})$ into a topological semigroup. Theorem 6 gives some equivalent conditions in order that (1) is a base for a topology $\mathfrak{T}^{\#}$ on $T$. In this case $\mathfrak{T}^{\#}$ obeys $\mathfrak{T}^{\#} \mid S \subseteq \subseteq$ and turns out to be the quotient topology corresponding to the mapping

$$
S \times \Sigma \rightarrow T:(a, \alpha) \rightarrow a \alpha^{-1} .
$$

Further, there is a necessary and sufficient condition (cf. Theorem 7$)$ that $\left(T, \cdot, \mathfrak{I}^{\#}\right)$ is a topological semigroup, and Theorem 8 gives a sufficient condition that $\left(G, \cdot, \mathfrak{T}^{\#} \mid G\right)$ is a topological group for a subgroup $G$ of $T$. Finally, we have a look at semigroups $(S, \cdot, \subseteq)$ satisfying the above property on translations using the results of $\S 6$.

We remark, that most of the statements of $[2, \S 3]$ concerning the group of quotients $T=Q(S, S)$ of a commutative semigroup $(S, \cdot, \subseteq)$ are covered by the second part of this paper. Here we mention the following result [2, Theorems 2, 4 and 8]: If all translations by elements $a \in S$ are open mappings in $(S, \cdot, \subseteq)$, then there exists a topology $\mathfrak{T}$ which makes the group $T=Q(S, S)$ into a topological group such that $\mathfrak{T} \mid S \subseteq \mathfrak{S}$ holds Such a topology can be obtained by a base (cf. (1))

$$
\left\{U V^{-1} \mid U, V \subseteq S \text { and open }\right\} .
$$

The same result is also stated by Paalman de Miranda [6, Theorem 3.2.14, p. 122], but even with $\mathfrak{T} \mid S=\mathfrak{S}$ instead of $\mathfrak{T} \mid S \subseteq \mathfrak{S}$. This sharpening fails to be true (the critical point in [6] is on page 123, line 12, where in general only $\alpha(U(a)) \subseteq$ $P(U(a) U(a) \times U(a))$ holds) as we shall show by examples in $\$ 4$. More precisely, for a semigroup $(S, \cdot, S)$ satisfying the assumptions of this theorem, the following situation concerning the group $T=Q(S, S)$ is possible: If $\mathfrak{T}$ is a topology on $T$ such that $(T, \cdot, \mathfrak{I})$ is a topological group (for instance, the topology $\mathfrak{T}$ regarded in [2] and [6]), then only $\mathfrak{I} \mid S \subset \mathfrak{S}$ is true. If $\mathfrak{T}$ is a topology on $T$ with $\mathfrak{T} \mid S=\subseteq$ (for instance, the topology of our Theorem 1 with $\Sigma=S)$, then $(T, \cdot, \mathfrak{T})$ can be a topological semigroup, but not a topological group.

We mention, that most of our results also hold for semitopological semigroups instead of topological semigroups. Moreover, there are applications to rings and semirings of quotients as well as to rings and semirings of differences (for these concepts cf. [10]). We expect to deal with this matter in a later publication.

The author is indebted to Professor A. D. Wallace for many stimulating discussions and acknowledges the support of the National Science Foundation (NSF GP-6505) and of the Deutsche Forschungsgemeinschaft.

2. Preliminaries. Let $S=(S, \cdot)$ be a semigroup and $\Sigma$ a subsemigroup of $S$. A semigroup of right quotients of $S$ with respect to $\Sigma$ is defined to be a semigroup 
$T=(T, \cdot)$ with the following properties: $T$ has an identity and contains $S$ as a subsemigroup, each $\alpha \in \Sigma$ has an inverse $\alpha^{-1} \in T$, and each element of $T$ is a right quotient $a \alpha^{-1}$ with $a \in S, \alpha \in \Sigma$. We recall (cf. [12]) that such a semigroup $T$ exists, if and only if, each $\alpha \in \Sigma$ is cancellable in $S$ and the condition

$q_{r}(S, \Sigma) \quad a \in S$ and $\alpha \in \Sigma$ implies $a \lambda=\alpha l$ for some $l \in S, \lambda \in \Sigma$, i.e. $a \Sigma \cap \alpha S \neq \varnothing$

holds. Then $T$ is, up to isomorphisms, uniquely determined by $S$ and $\Sigma$, and we write $T=Q_{r}(S, \Sigma) . T$ is governed by the rules

$$
\begin{gathered}
a \alpha^{-1}=b \beta^{-1} \Leftrightarrow \alpha \lambda=\beta l \text { and } a \lambda=b l \quad \text { for some } \lambda \in \Sigma \text { and } l \in S, \\
a \alpha^{-1} \cdot b \beta^{-1}=a t(\beta \tau)^{-1} \quad \text { with } b \tau=\alpha t, \quad \tau \in \Sigma, t \in S .
\end{gathered}
$$

Further, if $\Sigma^{*}$ denotes the subsemigroup of all elements of $S$ which have an inverse in $T=Q_{r}(S, \Sigma)$, then we have $Q_{r}(S, \Sigma)=Q_{r}\left(S, \Sigma^{*}\right)$ and $G^{*}=Q_{r}\left(\Sigma^{*}, \Sigma^{*}\right)$ is the unique maximal subgroup of $T$. We call $\Sigma^{*}$ a relatively maximal subsemigroup of right denominators of $S$, and $\Sigma^{*}$ is related to each $\Sigma$ for which $Q_{r}(S, \Sigma)=Q_{r}\left(S, \Sigma^{*}\right)$ holds by

$$
\xi^{*} \in \Sigma^{*} \Leftrightarrow \begin{aligned}
& \xi^{*} \text { left cancellable in } S \text { and } \\
& \xi^{*} x \in \Sigma \text { for some } x \in S
\end{aligned} \Rightarrow x \in \Sigma^{*} .
$$

Of course, (4) with $\Sigma=\Sigma^{*}$ is a necessary and sufficient condition for a subsemigroup of right denominators of $S$ to be relatively maximal.

Finally, if there are subsemigroups of right denominators in $S$ (i.e. if at least one $T=Q_{r}(S, \Sigma)$ exists), then there is a unique absolutely maximal subsemigroup of right denominators $\Sigma^{* *}$, containing all the others. Therefore $T^{* *}=Q_{r}\left(S, \Sigma^{* *}\right)$ is a semigroup of right quotients of $S$ such that $T^{* *} \supseteq T$ for all $T=Q_{r}(S, \Sigma)$. In particular, there exists a group of right quotients of $S$ if and only if $\Sigma^{* *}=S$. In this context we also refer to [10] and [11].

Now let $S=(S, \cdot)$ be a semigroup and let $\subseteq$ be a topology for $S$, which we regard as the family of open subsets of $S$. Then $S=(S, \cdot, \subseteq)$ is called a topological (semitopological) semigroup if the multiplication $a \cdot b$ is continuous in both variables $a, b$ together (in each variable $a, b$ separately). If $(S, \cdot)$ happens to be a group, then we call $(S, \cdot, \subseteq)$ a topological (semitopological) group if $(S, \cdot, \subseteq)$ is a topological (semitopological) semigroup and the mapping $a \rightarrow a^{-1}$ is continuous (here we differ from [3], where "semitopological group" is used for semitopological semigroups which are algebraically groups, i.e. without continuity for $a^{-1}$; cf. also the remark at the end of §3). Finally we do not assume any separation axiom unless otherwise stated. For concepts and propositions used without comment we refer to [1] and [5].

\section{A special topology for semigroups of right quotients.}

THEOREM 1. Let $S=(S, \cdot$, S) be a topological semigroup and let $T=(T, \cdot)$ $=Q_{r}(S, \Sigma)$ be a semigroup of right quotients of $S$ with respect to $\Sigma$. Then there 
exists a topology $\mathfrak{T}$ for $T$ with $\mathfrak{T} \mid S=\Im$ such that $T=(T, \cdot, \mathfrak{T})$ becomes a topological semigroup containing $S$ as an open subset, if and only if,

$$
U \in \mathbb{S} \text { implies } U \alpha \in \mathfrak{S} \text { and } \alpha U \in \mathfrak{S} \text { for each } \alpha \in \Sigma \text {. }
$$

Moreover, if $\mathfrak{B}$ is any base of $\Subset$, then the topology $\mathfrak{T}$ for $T$ with the properties above is defined by the base

$$
\mathfrak{B} \Sigma^{-1}=\left\{U \alpha^{-1} \mid U \in \mathfrak{B}, \alpha \in \Sigma\right\}
$$

and hence uniquely determined by $\subseteq$.

REMARK. The condition (5) states that all inner translations $\rho_{\alpha}: x \rightarrow x \alpha$, $\lambda_{\alpha}: x \rightarrow \alpha x$ of $S$ with $\alpha \in \Sigma$ are open mappings (from $S$ into $S$, briefly in $S$ ). Moreover, one can replace (5) by the formally weaker condition: If $x \in S$ and $\alpha \in \Sigma$, then

$$
\begin{aligned}
& \text { for each } U_{x} \text { exists } U_{x \alpha} \text { with } U_{x} \propto \supseteq U_{x \alpha}, \\
& \text { for each } U_{x} \text { exists } U_{\alpha x} \text { with } \alpha U_{x} \supseteq U_{\alpha x} \text {. }
\end{aligned}
$$

Here $U_{x}$ always denotes a set out of $\mathfrak{B}$ containing $x \in S$. We further mention that for each topology $\subseteq$ on $S$ which does not fulfill (5) there is a unique coarsest topology $\Im^{\prime}$ with $\varsigma^{\prime} \supset \subseteq$ obeying $(5)$, but $\left(S, \cdot, \Im^{\prime}\right)$ need not to be a topological semigroup without supplementary conditions (for a particular case $\mathrm{cf}$. [6, Theorem 3.2.13, p. 121]).

Proof. First we assume that there exists such a topology $\mathfrak{T}$ for $T$. Then all inner translations $\lambda_{\alpha}, \rho_{\alpha}$ of $T$ with $\alpha \in \Sigma$ are homeomorphisms of $T$ onto $T$ (cf. the similar proof in [3, p. 28] and hence open mappings in $T$. Since $S \in \mathfrak{T}$, their restrictions to $S$, i.e. the inner translations $\lambda_{\alpha}, \rho_{\alpha}$ of $S$ with $\alpha \in \Sigma$, are open mappings in $S$. Indeed, $U \in \mathfrak{S} \Rightarrow U \in \mathfrak{T} \Rightarrow U \alpha \in \mathfrak{I} \Rightarrow U \alpha \in \mathfrak{S}$, so we have (5). Now we shall use that each element of $T$ is a right quotient $a \alpha^{-1}$ with $a \in S, \alpha \in \Sigma$, to prove (6). Assume $a \alpha^{-1} \in V \in \mathfrak{T}$. Then $V \alpha \cap S \subseteq S$ is $\subseteq$-open and contains $a$, hence there exists $U_{a} \in \mathfrak{B}$ with $a \in U_{a} \subseteq V \alpha \cap S$ and we have

$$
a \alpha^{-1} \in U_{a} \alpha^{-1} \subseteq(V \alpha \cap S) \alpha^{-1} \subseteq V
$$

as we were to show.

Conversely, let $T=(T, \cdot)=Q_{r}(S, \Sigma)$ be any semigroup of right quotients of $S=(S, \cdot, \subseteq)$ and assume (5). We shall prove that (6) defines a base for a topology $\mathfrak{I}$ on $T$ satisfying our theorem. For the first suppose

$$
x \xi^{-1} \in U_{1} \alpha_{1}^{-1} \cap U_{2} \alpha_{2}^{-1} \text { with } U_{i} \in \mathfrak{B} .
$$

Then by $q_{r}(S, \Sigma)$ there are elements $l_{i} \in S, \lambda_{i} \in \Sigma$ with $\alpha_{i} \lambda_{i}=\xi l_{i}$ and from (7) we obtain $x l_{i} \in U_{i} \lambda_{i}(i=1,2)$. From (5) we have $U_{i} \lambda_{i} \in \mathfrak{S}$, and by the continuity and base properties of $\mathfrak{B}$ there is a common $U_{x} \in \mathfrak{B}$ with $U_{x} l_{i} \subseteq U_{i} \lambda_{i}$, from which

$$
x \xi^{-1} \in U_{x} \xi^{-1} \subseteq U_{1} \alpha_{1}^{-1} \cap U_{2} \alpha_{2}^{-1}
$$


follows. Hence $\mathfrak{B \Sigma ^ { - 1 }}$ is a base for a topology $\mathfrak{T}$. Clearly $\left\{U_{a} \alpha^{-1}\right\}$ is a base on $a \alpha^{-1}$, and $\mathfrak{T}$ does not depend on the choice of $\mathfrak{B}$, where the latter also may be concluded from our theorem after completing the proof.

In order to show that the multiplication in $(T, \cdot, \mathfrak{T})$ is continuous assume (cf. (3))

$$
a \alpha^{-1} \cdot b \beta^{-1}=(a t)(\beta \tau)^{-1} \in U_{a t}(\beta \tau)^{-1}, \quad b \tau=\alpha t .
$$

There exist $U_{a}, U_{t}$ with $U_{a} U_{t} \subseteq U_{a t}$, and since $b \tau=\alpha t \in \alpha U_{t} \in \mathbb{S}$ there is a $U_{b}$ with $U_{b} \tau \subseteq \alpha U_{t}$, from which $\alpha^{-1} U_{b} \subseteq U_{t} \tau^{-1}$ follows. But then we have already

$$
a \alpha^{-1} \cdot b \beta^{-1} \in U_{a} \alpha^{-1} U_{b} \beta^{-1} \subseteq U_{a} U_{t} \tau^{-1} \beta^{-1} \subseteq U_{a t}(\beta \tau)^{-1} \text {. }
$$

Next $\subseteq \supseteq \mathfrak{T} \mid S$ holds, since $\left\{U \alpha^{-1} \cap S\right\}$ is a base for $\mathfrak{I} \mid S$ and for each element $c \in U \alpha^{-1} \cap S$ there exists $U_{c}$ from $U_{c} \alpha \subseteq U$, which obeys $c \in U_{c} \subseteq U \alpha^{-1} \cap S$.

We remark that these three steps of the "if-part" of our proof also can be deduced from the Theorems 6 and 7 of $\S 6$. We shall sketch this way at the beginning of $\S 7$.

Finally, each $\subseteq$-open set $U \subseteq S$ is according to

$$
U=(U \alpha) \alpha^{-1}=\left(\bigcup U_{i}\right) \alpha^{-1}=\bigcup\left(U_{i} \alpha^{-1}\right) \in \mathfrak{T}, \quad U_{i} \in \mathfrak{B}
$$

also $\mathfrak{T}$-open, from which $S \in \mathfrak{T}$ and $\mathfrak{S} \subseteq \mathfrak{T} \mid S$ follow. This completes the proof of Theorem 1, from which the following two corollaries are immediate conclusions.

Corollary 1. Let $S=(S, \cdot, \subseteq)$ be a topological semigroup and let $\Sigma, \Sigma^{\prime}$ be different subsemigroups of $S$ such that $T=Q_{r}(S, \Sigma)=Q_{r}\left(S, \Sigma^{\prime}\right)$. Then (5) with respect to $\Sigma$ holds, if and only if, (5) with respect to $\Sigma^{\prime}$ is true. In this case, $\mathfrak{B \Sigma ^ { - 1 }}$ and $\mathfrak{B \Sigma ^ { \prime - 1 }}$ are bases for the same topology $\mathfrak{T}$ on $T$ described in Theorem 1.

In particular, in dealing with Theorem 1 one may choose $\Sigma$ as small as possible or as the relative maximal subsemigroup of right quotients $\Sigma^{*}$ corresponding to $\Sigma$, as it is convenient. For an illustration of this situation see the first example in $\S 4$.

Now let $T_{1}=Q_{r}\left(S, \Sigma_{1}\right)$ be a semigroup of right quotients of $S$ and let $T_{2}=$ $Q_{r}\left(T_{1}, \Theta\right)$ be a semigroup of right quotients of $T_{1}$. Then $T_{2}$ is also a semigroup of right quotients of $S$ with respect to a suitable subsemigroup $\Sigma_{2}$, i.e. $T_{2}=Q_{r}\left(S, \Sigma_{2}\right)$ (cf. [11]). Of course, $T_{2}$ is a subsemigroup of the maximal semigroup of right quotients $T^{* *}$ of $S$ described in $\S 2$.

Corollary 2. Let $S=\left(S, \cdot\right.$, S) be a topological semigroup and let $T_{1}, T_{2}$ be as above. Assume that $S$ satisfies (5) with respect to $\Sigma_{1}$. Then $S$ satisfies (5) with respect to $\Sigma_{2}$, if and only if, $T_{1}$ satisfies (5) with respect to $\Theta$. In this case, reaching $T_{2}$ in two steps or in one step and always applying Theorem 1 , the obtained topology $\mathfrak{I}_{2}$ for $T_{2}$ is the same.

Of course, in all these cases, (5) need not to be true with respect to the absolutely maximal subsemigroup of right denominators $\Sigma^{* *}$ of $S$. But one can prove: 
Corollary 3. Let $S=(S, \cdot$, S) be a topological semigroup for which a subsemigroup $\Sigma$ of right denominators obeying (5) exists. Then among all $\Sigma$ of this kind there is a unique maximal one, $\Sigma^{\prime \prime}$, so that $T^{\prime \prime}=Q_{r}\left(S, \Sigma^{\prime \prime}\right)$ is the unique maximal semigroup of right quotients of $S$ which possesses a topology $\mathfrak{T}^{\prime \prime}$ according to Theorem 1 .

TheOREM 2. Let $S=(S, \cdot, \subseteq)$ and $T=Q_{r}(S, \Sigma)=(T, \cdot, \mathfrak{T})$ be topological semigroups according to Theorem 1 . Then, if $(S, \Im)$ is a locally compact Hausdorff space, the same holds for $(T, \mathfrak{T})$.

Proof. Clearly the separation axiom $T_{2}$ (as well as some other topological properties, for instance connectedness) carries over from $\mathfrak{S}$ to $\mathfrak{T}$. Now let $a \alpha^{-1}$ be any element of $T$. According to (5) $S \alpha^{-1}$ is a locally compact subspace of $(T, \mathfrak{T})$. Hence there is an open set $U \subseteq S \alpha^{-1}, a \alpha^{-1} \in U$, with a compact closure $U^{\prime}$-all concepts used with respect to the subspace $S \alpha^{-1}$. But then clearly $U^{\prime}$ is also a compact neighborhood of $a \alpha^{-1}$ in $(T, \mathfrak{I})$ and this completes our proof.

COROLlaRY. If under the assumptions above $(T, \cdot)$ happens to be a group, then $(T, \cdot, \mathfrak{T})$ is a topological group.

REMARKs. All statements above are also true for semitopological semigroups and semitopological groups (and even, more generally, if one only looks at continuity of the multiplication from one side). In this context, and with respect to the last corollary, we recall that we use the term "semitopological group" to include the continuity of the mapping $a \rightarrow a^{-1}$. We may mention that there exist semitopological groups in our sense which are not topological groups. For instance one can establish the group of positive reals with a topology such that $a \rightarrow a^{-1}$ is continuous, $a \cdot b$ is continuous with respect to each variable separately, but not with respect to both variables together.

\section{Examples.}

1. The group $(T, \cdot)$ of positive rationals (reals) with the usual multiplication is the group of quotients of the semigroup $(S, \cdot)$, where $S$ is any open interval $(0, t)$, $t \leqq 1$. Here we have $T=Q(S, S)=Q(S, \Sigma)$ for each subsemigroup $\Sigma$ of $S$. Taking the usual topology on $S$, Theorem 1 (and also Theorem 2) is applicable and gives the usual topology on $T$. Moreover, this topology is the unique one for the positive rationals (reals) making the multiplication continuous and preserving the usual topology and openness of at least one interval $(0, t)$.

2. (a) Let $(T, \cdot)$ be again the group of positive rationals and let $S=Q(N, \theta)$ be any semigroup of quotients of the naturals $N$ such that $N \subset S \subset T$. We assume the subsemigroup $\theta$ of $N$ to be relatively maximal, hence $\theta$ is generated by all primes $p$ with $p^{-1} \in S$ (cf. $\S 2$ ). Because of $S \subset T$ there is at least one prime $q$, such that $q^{-1} \notin S$, or equivalently, $q \notin \theta$. Clearly, $T=Q(S, S)$, and we denote the usual topology on $T$ by $\Re$. For $\subseteq=\Re \mid S,(S, \cdot, \subseteq)$ is a topological semigroup.

(b) $(S, \cdot, \subseteq)$ does not satisfy (5) with respect to $\Sigma=S$. We consider a set

$$
U=\{a|\alpha| a \in N, \alpha \in \theta,|a| \alpha-1 \mid<\varepsilon \text { for some } \varepsilon>0\}
$$


contained in $\Im=\Re \mid S$. Let $q$ be a prime not contained in $\theta$. We are going to prove $q U \notin \Im$. Let us suppose the contrary, then there exists a $V \in \Xi$ such that $q \in V \subseteq q U$. For a prime $p \in \theta$ one has $\left(q p^{n}+1\right) / p^{n} \in S$, hence $\left(q p^{n}+1\right) / p^{n} \in V$ if $n$ is large enough. But $\left(q p^{n}+1\right) / p^{n}=q a / \alpha \in q U$ leads to $q\left|\alpha\left(q p^{n}+1\right) \Rightarrow q\right| \alpha \Rightarrow q \in \theta$, a contradiction completing our proof.

(c) Now we endow $S$ with a topology $\mathfrak{S}^{\prime} \supset \mathbb{S}$, such that $\left(S, \cdot, \mathbb{S}^{\prime}\right)$ is a topological semigroup which satisfies (5) with respect to $\Sigma=S$. According to [6, Theorem 3.2.13,

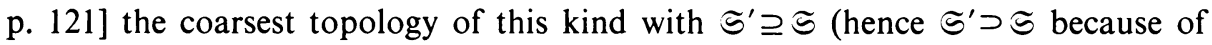
(b)) is obtained using the family

$$
\{s U|s \in S, U \in \mathfrak{S}=\Re| S \text { such that } 1 \in U\}
$$

as a base for $\mathbb{S}^{\prime}$. Note that $\subseteq$ and $\mathbb{S}^{\prime}$ have the same neighborhoods at 1 . Here we have counterexamples to Theorem 3.2.14 of [6] as announced in the introduction. We shall prove: There is no topology $\mathfrak{I}$ on $T=Q(S, S)$ such that $(T, \cdot, \mathfrak{T})$ is a topological group and $\mathfrak{I} \mid S=\mathbb{S}^{\prime}$ holds.

Let us suppose that such a topology $\mathfrak{I}$ would exist. Let $V$ be any set with $1 \in V \in \mathfrak{T}$. Then there are $V_{1}, V_{2} \in \mathfrak{T}$, both containing 1 , such that $V_{1} V_{2}^{-1} \subseteq V$. Because of $\mathfrak{T} \mid S=\mathbb{S}^{\prime}, V_{1} \cap S$ and $V_{2} \cap S$ contain all $x / \xi \in S$ out of suitable intervals containing 1 , hence $(x / \xi)(\eta / y) \in V$ for all $x / \xi, y / \eta \in S$ with $|x / \xi-1|<\varepsilon$, $|\eta| y-1 \mid<\varepsilon$ for a suitable $\varepsilon>0$. Each $a / b \in T$ can be written in the form $a / b=$ $(a z / \xi)(\xi / b z)$. Since $S$ is dense in $T$, a straightforward calculation shows that for all $a / b$ with $|a| b-1 \mid<\varepsilon / 2$ one can find $z$ and $\xi$ such that $a z / \xi=x / \xi$ with $|x / \xi-1|<\varepsilon$, $\xi / b z=\xi / y$ with $|\xi| y-1 \mid<\varepsilon$. So any $V$ with $1 \in V \in \mathfrak{T}$ contains an interval $U$ with $1 \in U \in \Re$, from which follows $\mathfrak{T} \subseteq \Re$. But this gives $\mathfrak{I}\left|S=\Im^{\prime} \subseteq \mathfrak{R}\right| S=\mathfrak{S}$, a contradiction to $\mathfrak{S}^{\prime} \supset \mathfrak{S}$.

We may remark, that according to Theorem 1 there is a topology $\mathfrak{I}^{\prime}$ on $T$, such that $\mathfrak{T}^{\prime} \mid S=\mathbb{S}^{\prime}$ and $S \in \mathfrak{T}^{\prime}$, which makes $\left(T, \cdot, \mathfrak{T}^{\prime}\right)$ into a topological semigroup. By the last result, $g \rightarrow g^{-1}$ cannot be continuous with respect to $\mathfrak{I}^{\prime}$, hence (5), but not $F(S, S)$ hold for $\left(S, \cdot, S^{\prime}\right)$ (cf. $\left.\S 5\right)$. On the other hand, there is a topology $\mathfrak{T}^{\prime \prime}$ on $T$ such that $\left(T, \cdot, \mathfrak{T}^{\prime \prime}\right)$ is a topological group and $\mathfrak{T}^{\prime \prime} \mid S \subseteq \Im^{\prime}$, hence $\mathfrak{I}^{\prime \prime} \mid S \subset \mathfrak{S}^{\prime}$, for instance the usual topology $\Re$.

5. Continuity of $g \rightarrow g^{-1}$ for the topology considered in $\S 3$. Let $S=(S, \cdot, \subseteq)$ be a topological semigroup and $T=(T, \cdot)=Q_{r}(S, \Sigma)$ a semigroup of right quotients of $S$. We regard subgroups $G=(G, \cdot)$ of $T$ containing $\Sigma$ and define $\Sigma^{\prime}=G \cap S$. Clearly one has $\Sigma \subseteq \Sigma^{\prime} \subseteq \Sigma^{*}, Q_{r}(S, \Sigma)=Q_{r}\left(S, \Sigma^{\prime}\right)=Q_{r}\left(S, \Sigma^{*}\right)$, and

$$
G=Q_{r}\left(\Sigma^{\prime}, \Sigma\right)=Q_{r}\left(\Sigma^{\prime}, \Sigma^{\prime}\right) \subseteq G^{*}=Q_{r}\left(\Sigma^{*}, \Sigma\right)=Q_{r}\left(\Sigma^{*}, \Sigma^{*}\right),
$$

with $\Sigma^{*}$ and $G^{*}$ as defined in $\S 2$. We define with respect to $(S, \cdot, \subseteq)$ the following conditions:

$F_{r}\left(\Sigma, \Sigma^{\prime}\right)$ Suppose $a \in U \in \mathfrak{S}$ and $\alpha \in \Sigma$. Then there exists $V_{\alpha} \in \mathfrak{S}$ such that $a \alpha \in U \alpha^{\prime}$ for each $\alpha^{\prime} \in V_{\alpha} \cap \Sigma^{\prime}$.

$F_{l}\left(\Sigma, \Sigma^{\prime}\right)$ dually, i.e., $\alpha a \in \alpha^{\prime} U$ instead of $a \alpha \in U \alpha^{\prime}$. 
Obviously one can choose the same $V_{\alpha} . F_{r}(S)=F_{r}(S, S)$ and $F_{l}(S)=F_{l}(S, S)$ together are the condition $F$ introduced by Rothman [7, Definition 1], already mentioned in the introduction.

THEOREM 3. Let $S=\left(S, \cdot\right.$, s) and $T=Q_{r}(S, \Sigma)$ be given and assume (5), i.e., the existence of the topology $\mathfrak{I}$ on $T$ as considered in Theorem 1 . Let $G$ be a subgroup of $T, G \supseteq \Sigma$, and put $\Sigma^{\prime}=G \cap S$. Then $(G, \cdot, \mathfrak{T} \mid G)$ is a topological group if, and only if, $F_{r}\left(\Sigma, \Sigma^{\prime}\right)$ holds.

Proof. First we assume $(G, \cdot, \mathfrak{I} \mid G)$ to be a topological group. Let $a \in U \in \mathfrak{S}$ and $\alpha \in \Sigma$ be given. Hence $a \alpha \alpha^{-1} \in U \in \mathfrak{S}$ and by Theorem $1 U \in \mathfrak{T}$. There exists a $V^{\prime}$ with $\alpha^{-1} \in V^{\prime} \in \mathfrak{T}$ such that $a \alpha \alpha^{-1} \in a \alpha V^{\prime} \subseteq U$. From $\alpha^{-1} \in V^{\prime} \cap G \in \mathfrak{T} \mid G$ it follows the existence of a $V \in \mathfrak{T}$ such that $\alpha \in V \cap G \in \mathfrak{T} \mid G$ and $(V \cap G)^{-1} \subseteq V^{\prime} \cap G$, hence

$$
a \alpha \alpha^{-1} \in a \alpha(V \cap G)^{-1} \subseteq U .
$$

This proves $a \alpha \in U \alpha^{\prime}$ for each $\alpha^{\prime} \in V \cap G$. Define $V_{\alpha}=V \cap S$, then $V_{\alpha} \in \Im$ and $V_{\alpha} \cap \Sigma^{\prime} \subseteq V \cap G$, from which we obtain $F_{r}\left(\Sigma, \Sigma^{\prime}\right)$.

Conversely, we have to show that $g \rightarrow g^{-1}$ is continuous for each $g=a \alpha^{-1} \in G$, with respect to $\mathfrak{I} \mid G$. Let $U \beta^{-1} \cap G$ be any set out of $\mathfrak{I} \mid G$, containing $g^{-1}$. Then we have $g^{-1}=b \beta^{-1}$ with $b \in U \in \subseteq$ and $\beta \in \Sigma$. By $F_{r}\left(\Sigma, \Sigma^{\prime}\right)$ there is a $V_{\beta} \in \subseteq$ such that $b \beta \in U \beta^{\prime}$ for all $\beta^{\prime} \in V_{\beta} \cap \Sigma^{\prime}=V_{\beta} \cap G$. This means $b \beta \beta^{\prime-1} \in U$, hence

$$
b \beta\left(V_{\beta} \cap G\right)^{-1} \subseteq U .
$$

From $b \in G$ follows $\beta^{-1} b^{-1} \in G=Q_{r}\left(\Sigma^{\prime}, \Sigma\right)$, hence $\beta^{-1} b^{-1}=c \gamma^{-1}$ with suitable elements $c \in \Sigma^{\prime}, \gamma \in \Sigma$. Looking for a neighbourhood of $g=\beta b^{-1}$ we obtain

$$
\beta\left(V_{\beta} \cap G\right) \beta^{-1} b^{-1}=\left(\beta V_{\beta} c \cap G\right) \gamma^{-1}=\left(\beta V_{\beta} c\right) \gamma^{-1} \cap G \in \mathfrak{T} \mid G,
$$

because of $V_{\beta} \in \mathfrak{S} \Rightarrow \beta V_{\beta} \in \mathfrak{S} \Rightarrow \beta V_{\beta} c \in \mathbb{S}$ (for the latter we use $c \in \Sigma^{\prime}$ and Corollary 1 of Theorem 1). Now we have

$$
g \in \beta\left(V_{\beta} \cap G\right) \beta^{-1} b^{-1} \in \mathfrak{T} \mid G
$$

and according to (8)

$$
g^{-1} \in\left(\beta\left(V_{\beta} \cap G\right) \beta^{-1} b^{-1}\right)^{-1}=b \beta\left(V_{\beta} \cap G\right)^{-1} \beta^{-1} \subseteq U \beta^{-1} \cap G,
$$

which proves the continuity of $g \rightarrow g^{-1}$ in $(G, \cdot, \mathfrak{I} \mid G)$.

In the same way, but with some more technical effort in the second part of the proof, one can show that Theorem 3 also holds with $F_{l}\left(\Sigma, \Sigma^{\prime}\right)$ instead of $F_{r}\left(\Sigma, \Sigma^{\prime}\right)$. From this follows the first part of the following corollary; the second is a direct conclusion of our theorem.

COROLlaRY. Under the assumptions of Theorem $3, F_{r}\left(\Sigma, \Sigma^{\prime}\right)$ and $F_{l}\left(\Sigma, \Sigma^{\prime}\right)$ are equivalent for each $\Sigma^{\prime}=G \cap S \supseteq \Sigma$.

If further $T=Q_{r}(S, \Sigma)=Q_{r}\left(S, \Sigma_{1}\right)$ and $G_{1}$ is a subgroup of $T$ such that $\Sigma^{\prime}=G \cap S$ $\supseteq \Sigma_{1}^{\prime}=G_{1} \cap S \supseteq \Sigma_{1}$, then $F_{r}\left(\Sigma, \Sigma^{\prime}\right)$ imply $F_{r}\left(\Sigma_{1}, \Sigma_{1}^{\prime}\right)$. 
Observe, that the last statement with $G=G_{1}$ is a pendant to Corollary $1, \S 3$. Also the Corollaries 2 and 3 have similar generalizations concerning (5) and the condition $F_{r}$ or $F_{l}$. The next theorem is the announced generalization of [7, Theorem 2.1].

THEOREM 4. Let $S=(S, \cdot, \subseteq)$ be a topological semigroup which possesses a group $T=Q_{r}(S, S)$ of right quotients. Then there exists a topology $\mathfrak{I}$ on $T$ such that $(T, \cdot, \mathfrak{T})$ is a topological group, $\mathfrak{T} \mid S=\mathfrak{S}$ and $S \in \mathfrak{T}$, if and only if, (5) with respect to $S$ and $F_{r}(S)$ hold. In this case there is exactly one topology $\mathfrak{I}$ of this kind. Moreover, $F_{r}(S)$ and $F_{l}(S)$ together imply $(5)$.

Note that the equivalence of $F_{r}(S)$ and $F_{l}(S)$ stated in the corollary above depends on (5).

Proof. Combining Theorem 1 and Theorem 3 for $\Sigma=S$ and $G=T$, one obtains all statements except the last one. We are going to prove $U \in \Im \Rightarrow U a \in \subseteq$ for each $a \in S$, and we regard an element $x=u a \in U a$. From $F_{l}(S)$ we have $a u \in a^{\prime} U$ for all $a^{\prime}$ contained in a suitable $V_{a} \in \Im$. From $F_{r}(S)$ it follows $a x \in V_{a} x^{\prime}$ for all $x^{\prime}$ contained in some $W_{x} \in \subseteq$ S. Hence for each $x^{\prime} \in W_{x}$ there is an $a^{\prime} \in V_{a}$ with $a x=a^{\prime} x^{\prime}$, and for this $a^{\prime}$ exists an $u^{\prime} \in U$ such that $a u=a^{\prime} u^{\prime}$ holds. Therefore

$$
a^{\prime} x^{\prime}=a x=a u a=a^{\prime} u^{\prime} a,
$$

from which follows $x^{\prime}=u^{\prime} a$, i.e., $W_{x} \subseteq U a$ (cf. [7, Lemma 1.3]). The proof for $U \in \mathfrak{S} \Rightarrow a U \in \mathbb{S}$ is just the same.

6. The general case. Again $S=(S, \cdot, \subseteq)$ means a topological semigroup and $T=(T, \cdot)=Q_{r}(S, \Sigma)$ a semigroup of right quotients of $S$. We are going to investigate topologies $\mathfrak{I}$ for $T$ such that $(T, \cdot, \mathfrak{I})$ is a topological semigroup and $\mathfrak{I} \mid S \subseteq \subseteq$ holds. According to the next theorem we have to consider certain topologies $\mathfrak{Z}$ on $\Sigma$, which may differ from $\subseteq \mid \Sigma$ (for instance, $\S 3$ will be covered taking the discrete topology for $\mathfrak{s})$.

From the algebraic structure of $T$ we have the mapping

$$
P: S \times \Sigma \rightarrow T \text { defined by }(a, \alpha) \rightarrow a \alpha^{-1},
$$

which will be useful in our topological considerations. We may denote the product topology in $S \times \Sigma$ by $\subseteq \times \mathfrak{g}$, although $\subseteq \times \mathfrak{g}$ is only a base for this topology. We recall, that the quotient topology $\mathfrak{T}^{\#}$ for $T$ (with respect to (9) and $\mathfrak{S} \times \mathfrak{S}$ ) is the largest topology for $T$ such that

$$
P:(S \times \Sigma, \mathfrak{S} \times \mathfrak{S}) \rightarrow\left(T, \mathfrak{T}^{\#}\right)
$$

is continuous; as we always have the same mapping (9) we use $\mathfrak{I} \#(\subseteq \times \mathfrak{s})$ to determine this topology, depending on the choice of $\mathfrak{S} \times \mathfrak{G}$.

THEOREM 5. Let $S=(S, \cdot, S)$ and $T=Q_{r}(S, \Sigma)$ be given, and let $\mathfrak{T}$ be any topology for $T$ with $\mathfrak{I} \mid S \subseteq \mathfrak{S}$, such that $(T, \cdot, \mathfrak{I})$ is a topological semigroup. Then there exists 
a topology $\mathfrak{s}$ on $\Sigma$ which makes $(\Sigma, \cdot, \mathfrak{g})$ into a topological semigroup and has the property that each $\mathfrak{T}$-open subset $V \subseteq T$ is a union of sets out of the family

$$
\mathfrak{B}(\mathfrak{S}, \mathfrak{s})=\left\{U \Omega^{-1} \mid U \in \mathfrak{S}, \Omega \in \mathfrak{s}\right\} .
$$

Therefore the mapping

$$
P:(S \times \Sigma, \mathfrak{S} \times \mathfrak{Z}) \rightarrow(T, \mathfrak{T})
$$

is continuous, hence the quotient topology $\mathfrak{T}^{\#}(\mathfrak{S} \times \mathfrak{S})$ on $T$ satisfies $\mathfrak{T} \subseteq \mathfrak{T} \#(\mathfrak{S} \times \mathfrak{I})$, where equality holds if (11) is open.

Proof. We define $\mathfrak{b}$ to be the family of sets

$$
\Omega=\left(V \cap \Sigma^{-1}\right)^{-1}=(V \cap G)^{-1} \cap \Sigma
$$

for all $V \in \mathfrak{I}$, where $G$ is any subgroup of $T$ containing $\Sigma$. It is easily checked that the equality in (12) holds independent on the choice of $G$. Moreover, for each $\alpha \in \Sigma$ one has

$$
\alpha \in \Omega \Leftrightarrow \alpha^{-1} \in V .
$$

It is straightforward to prove that $\mathfrak{b}$ is a base for a topology $\mathfrak{s}$ on $\Sigma$. We show that $(\Sigma, \cdot, \mathfrak{g})$ is a topological semigroup. Suppose $\alpha \beta$ is contained in $\Omega$ as in (12). Then $\beta^{-1} \alpha^{-1} \in V$ and there are $V_{1}, V_{2} \in \mathfrak{I}$ such that $\beta^{-1} \in V_{1}, \alpha^{-1} \in V_{2}, V_{1} V_{2} \subseteq V$. From

we obtain

$$
\left(V_{1} \cap \Sigma^{-1}\right)\left(V_{2} \cap \Sigma^{-1}\right) \subseteq V_{1} V_{2} \cap \Sigma^{-1} \subseteq V \cap \Sigma^{-1}
$$

$$
\alpha \beta \in\left(V_{2} \cap \Sigma^{-1}\right)^{-1}\left(V_{1} \cap \Sigma^{-1}\right)^{-1} \subseteq\left(V \cap \Sigma^{-1}\right)^{-1}=\Omega
$$

as we were to prove. Now suppose $a \alpha^{-1} \in V \in \mathfrak{T}$. Then there are $V_{a}, V_{\alpha^{\prime}} \in \mathfrak{T}$ such that $a \in V_{a}, \alpha^{-1} \in V_{\alpha^{\prime}}, V_{a} V_{\alpha^{\prime}} \subseteq V$. By (12), $V_{\alpha^{\prime}}$ defines an $\Omega_{\alpha} \in \mathfrak{b}$ with $\Omega_{\alpha}^{-1} \subseteq V_{\alpha^{\prime}}$, and for $U_{a}=V_{a} \cap S \in \subseteq$ we obtain

$$
a \alpha^{-1} \in U_{a} \Omega_{\alpha}^{-1} \subseteq V_{a} V_{\alpha^{\prime}} \subseteq V .
$$

Hence each $V \in \mathfrak{T}$ is a union of suitable sets contained in $\mathfrak{B}(\mathfrak{S}, \mathfrak{b}) \subseteq \mathfrak{B}(\mathfrak{S}, \mathfrak{\mathfrak { B }})$. From (9) follows

$$
P(U \times \Omega)=U \Omega^{-1},
$$

hence (11) is continuous and our theorem is proved.

SUPPLEMENT. If, in particular, there is one subgroup $G$ of $T, G \supseteq \Sigma$, such that $(G, \cdot, \mathfrak{I} \mid G)$ is a topological group, then the topology $\mathfrak{s}$ on $\Sigma$ defined by (12) coincides

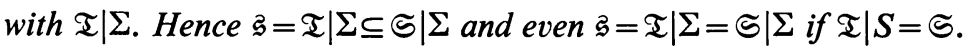

Proof. If $(G, \cdot, \mathfrak{T} \mid G)$ is a topological group, from $\mathfrak{I} \mid G=\{V \cap G \mid V \in \mathfrak{I}\}$ it follows $\mathfrak{I} \mid G=\left\{(V \cap G)^{-1} \mid V \in \mathfrak{I}\right\}$. Therefore (12) defines the topology

$$
\mathfrak{T}|G| \Sigma=\mathfrak{T} \mid \Sigma \text { on } \Sigma \text {. }
$$


We remark that the sets out of $(10)$ need not be $\mathfrak{I}$-open, moreover, $\mathfrak{B}(\mathfrak{S}, \mathfrak{Z})$ need not be a base for a topology on $T$. If the latter is the case, $\mathfrak{B}(\mathfrak{S}, \mathfrak{I})$ is a base for $\mathfrak{T}^{\#}(\mathfrak{S} \times \mathfrak{S}) \supseteq \mathfrak{T}$. This will be a conclusion from the next theorem, where we deal with this question under the following point of view: We intend to supply $T$ with suitable topologies. So we do not suppose that there exists such a topology as assumed in Theorem 5 , and use $(10)$ to define a family $\mathfrak{B}(\mathfrak{S}, \mathfrak{s})$ starting with $\mathfrak{S}$ and any topology $\mathfrak{s}$ on $\Sigma$.

TheOREM 6. Take $S=(S, \cdot, \subseteq)$ and $T=(T, \cdot)=Q_{r}(S, \Sigma)$ as above and let $\mathfrak{s}$ be any topology for $\Sigma$ such that $(\Sigma, \cdot, \mathfrak{g})$ is a topological semigroup. Then the following statements are equivalent:

(i) The mapping $P:(S \times \Sigma, \mathfrak{S} \times \mathfrak{I}) \rightarrow\left(T, \mathfrak{T}^{\#}(\mathfrak{S} \times \mathfrak{S})\right)$ is open.

(ii) $P^{-1}(P(U \times \Omega))$ is open for any $U \in \mathfrak{S}, \Omega \in \mathfrak{S}$.

(iii) $\mathfrak{B}(\mathfrak{S}, \mathfrak{g})$ in $(10)$ is a base for a topology on $T$.

(iv) $\mathfrak{B}(\mathfrak{S}, \mathfrak{g})$ is a base for $\mathfrak{T} \#(\mathfrak{S} \times \mathfrak{g})$.

(v) If $x \xi^{-1} \in U \Omega^{-1} \in \mathfrak{B}(\mathfrak{S}, \mathfrak{s})$, then there are $U_{x}, \Omega_{\xi}$ with $x \in U_{x} \in \mathfrak{S}, \xi \in \Omega_{\xi} \in \mathfrak{S}$ such that $U_{x} \Omega_{\xi}^{-1} \subseteq U \Omega^{-1}$.

If this is the case, then $\mathfrak{T}^{\#}(\mathfrak{S} \times \mathfrak{g}) \mid S \subseteq \mathfrak{S}$.

REMARK. Note that (v) establishes a kind of continuity for $x \xi^{-1}=a \alpha^{-1}$ (or $\xi l=\alpha \lambda, x l=a \lambda)$ in the sense that for given $U_{a}, \Omega_{\alpha}$ there are $U_{x}, \Omega_{\xi}$ such that for $x^{\prime} \in U_{x}, \xi^{\prime} \in \Omega_{\xi}$ there are $a^{\prime} \in U_{a}, \alpha^{\prime} \in \Omega_{\alpha}$ with $x^{\prime} \xi^{\prime-1}=a^{\prime} \alpha^{\prime-1}$ (or $\xi^{\prime} l^{\prime}=\alpha^{\prime} \lambda^{\prime}$, $\left.x^{\prime} l^{\prime}=a^{\prime} \lambda^{\prime}\right)$.

Proof. We shall prove (i) $\Rightarrow$ (ii) $\Rightarrow$ (v) $\Rightarrow$ (iii) $\Rightarrow$ (iv) $\Rightarrow$ (i). The first step is clear. For the second suppose $x \xi^{-1} \in U \Omega^{-1} \in \mathfrak{B}(\mathfrak{S}, \mathfrak{I})$. Then using (13) we have $(x, \xi) \in P^{-1}(P(U \times \Omega))$ and by (ii) there are $U_{x}, \Omega_{\xi}$ with $(x, \xi) \in U_{x} \times \Omega_{\xi} \subseteq$ $P^{-1}(P(U \times \Omega))$, from which (v) follows. Now we assume (v). Then it is easily seen that for

$$
x \xi^{-1} \in U_{1} \Omega_{1}^{-1} \cap U_{2} \Omega_{2}^{-1}, \quad U_{i} \Omega_{i}^{-1} \in \mathfrak{B}(\Im, \mathfrak{s})
$$

there exists $U_{x} \Omega_{\mathfrak{\xi}}^{-1} \in \mathfrak{B}(\mathfrak{S}, \mathfrak{g})$ with

$$
x \xi^{-1} \in U_{x} \Omega_{\xi}^{-1} \subseteq U_{1} \Omega_{1}^{-1} \cap U_{2} \Omega_{2}^{-1},
$$

which proves (iii). Denote the corresponding topology on $T$ by $\mathfrak{T}$. For the step (iii) $\Rightarrow$ (iv) we have to show $\mathfrak{T}=\mathfrak{T}^{\#}(\mathfrak{S} \times \mathfrak{g})$. For each $W \in \mathfrak{T}^{\#}(\mathfrak{S} \times \mathfrak{I})$ the set $P^{-1}(W)$ is open, hence $P^{-1}(W)$ is a union of suitable sets $U \times \Omega$. Therefore $P\left(P^{-1}(W)\right)=W$ is a union of sets $U \Omega^{-1}$, which proves $W \in \mathfrak{T}$. So we have $\mathfrak{T} \#(\mathfrak{S} \times \mathfrak{I}) \subseteq \mathfrak{T}$. Since the mapping $P:(S \times \Sigma, \mathfrak{S} \times \mathfrak{S}) \rightarrow(T, \mathfrak{T})$ clearly is continuous, it follows $\mathfrak{T}^{\#}(\mathfrak{S} \times \mathfrak{S})=\mathfrak{T}$. Finally (iv) implies (i), because $\{U \times \Omega\}$ is just the base considered in $S \times \Sigma$.

For the last statement suppose $c \in U \Omega^{-1} \cap S$. From $c=a \alpha^{-1}, a \in U, \alpha \in \Omega$ we obtain $c \alpha=a \in U$. Therefore we have an $U_{c} \in \subseteq$ such that $U_{c} \alpha \subseteq U$, hence

$$
c \in U_{c} \subseteq U \alpha^{-1} \subseteq U \Omega^{-1} \text { and } c \in U_{c} \in U \Omega^{-1} \cap S \text {, }
$$

which proves $\mathfrak{T} \#(\mathfrak{S} \times \mathfrak{g}) \mid S \subseteq \mathfrak{S}$. 
We remark, that the proof of the equivalence of (i)-(v) did not make use of any continuity of multiplication. Only the last statement depends on the left-sided continuity in $(S, \cdot, \mathbb{S})$.

Moreover, for given $(S, \cdot, \subseteq)$ and $T=Q_{r}(S, \Sigma)$, the validity of (i)-(v) clearly depends on the choice of $\Sigma$ and $\mathfrak{s}$. Even in the case $T=Q_{r}\left(S, \Sigma_{1}\right)=Q_{r}\left(S, \Sigma_{2}\right)$, $S \supseteq \Sigma_{1} \supseteq \Sigma_{2}, \mathfrak{I}_{2}=\mathfrak{I}_{1} \mid \Sigma_{2}$ one merely has $\mathfrak{T}^{\#}\left(\mathfrak{S} \times \mathfrak{I}_{1}\right) \subseteq \mathfrak{T}^{\#}\left(\subseteq \times \mathfrak{I}_{2}\right)$, but (i)-(v) may be satisfied only by one of these topologies on $T$. For example, take $(S, \cdot, \subseteq)$ and $T=Q(S, S)$ as in $\S 4,2($ a), and suppose for simplicity that only one prime $q$ is not contained in $\theta$. With $\Sigma_{1}=S, \Sigma_{2}=\left\{q, q^{2}, \ldots\right\}, \mathfrak{g}_{1}=\varsigma \mid \Sigma_{1}=\varsigma$ and $\mathfrak{g}_{2}=\mathfrak{g}_{1}\left|\Sigma_{2}=\varsigma\right| \Sigma_{2}$ we have the situation just regarded, and $\mathfrak{S}_{1}$ satisfies (i)-(v) (in fact, $\mathfrak{I} \#\left(\mathfrak{S} \times \mathfrak{g}_{1}\right)$ is the usual topology $\Re$ on $T$ ). But $\mathfrak{S}_{2}$ is the discrete topology on $\Sigma_{2}$ and

$$
\left\{U \alpha^{-1} \mid U \in \widetilde{S}, \alpha \in \Sigma_{2}\right\}
$$

is not a base for a topology on $T$ (this would imply (5), which is disproved in $\S 4$, 2(b)); hence $\mathfrak{S}_{2}$ does not satisfy (i)-(v).

Now suppose that for $(S, \cdot, \subseteq), T=Q_{r}(S, \Sigma)$ and a topology $\mathfrak{s}$ on $\Sigma$ the statements (i)-(v) of Theorem 6 are fulfilled. Even in these cases $(T, \cdot, \mathfrak{T} \#(\mathfrak{S} \times \mathfrak{s}))$ need not be a topological semigroup. This depends essentially on the fact that the mapping $P$ in (9) is a homomorphism from $S \times \Sigma$ onto $T$ only if $\Sigma$ is in the centre of $S$. In general, there is a natural supplementary assumption necessary and sufficient.

THEOREM 7. Let $S=(S, \cdot, \mathfrak{S})$ and $T=Q_{r}(S, \Sigma)$ be given and let $(\Sigma, \cdot, \mathfrak{I})$ be a topological semigroup for a topology $\mathfrak{s}$ satisfying $(\mathrm{i})-(\mathrm{v})$. Then $(T, \cdot, \mathfrak{T} \#(\mathfrak{S} \times \mathfrak{S}))$ is a topological semigroup, if and only if, the following condition holds:

$$
\begin{aligned}
& \text { If } \alpha^{-1} b \in U \Omega^{-1} \in \mathfrak{B}(\mathfrak{S}, \mathfrak{S}) \text {, then there are } U_{b}, \Omega_{\alpha} \text { with } b \in U_{b} \in \mathbb{S} \text {, } \\
& \alpha \in \Omega_{\alpha} \in \mathfrak{S} \text { such that } \Omega_{\alpha}^{-1} U_{b} \subseteq U \Omega^{-1} \text {. }
\end{aligned}
$$

Remark. Clearly (14) is a kind of continuity for the condition $q_{r}(S, \Sigma)$ in the following sense: Assume $\alpha t=b \tau$ (or $\left.\alpha^{-1} b=t \tau^{-1}\right)$ and let $U_{t}, \Omega_{\tau}$ be given. Then there exist $U_{b}, \Omega_{\alpha}$ such that for $b^{\prime} \in U_{b}, \alpha^{\prime} \in \Omega_{\alpha}$ there are $t^{\prime} \in U_{t}, \tau^{\prime} \in \Omega_{\tau}$ with $\alpha^{\prime} t^{\prime}=b^{\prime} \tau^{\prime}$.

SUPPLEMENT. If in particular $\Sigma$ is in the centre of $S$, then (14) coincides with (v), hence in this case each of the properties (i)-(v) is necessary and sufficient for $\left(T, \cdot, \mathfrak{I}^{\#}(\mathfrak{S} \times \mathfrak{S})\right)$ being a topological semigroup.

Proof. First we assume (14) and consider (cf. (3))

$$
a \alpha^{-1} \cdot b \beta^{-1}=a t(\beta \tau)^{-1} \in U_{a t} \Omega_{-\bar{\beta}^{-1}}^{1}, \quad b \tau=\alpha t .
$$

Then there are $U_{a}, U_{t}, \Omega_{\beta}, \Omega_{\tau}$ with

$$
U_{a} U_{t} \subseteq U_{a t} \text { and } \Omega_{\beta} \Omega_{\tau} \subseteq \Omega_{\beta \tau} \text {, i.e. } \Omega_{\tau}^{-1} \Omega_{\beta}^{-1} \subseteq \Omega_{\beta \tau}^{-1} \text {. }
$$

Moreover, for $U_{t}$ and $\Omega_{\tau}$ there are by assumption $U_{b}, \Omega_{\alpha}$ satisfying (14), so that we have

$$
U_{a} \Omega_{\alpha}^{-1} U_{b} \Omega_{\beta}^{-1} \subseteq U_{a} U_{t} \Omega_{\tau}^{-1} \Omega_{\beta}^{-1} \subseteq U_{a t} \Omega_{\beta \tau}^{-1}
$$


which proves continuity for multiplication. Conversely, let

$$
\alpha^{-1} b=t \tau^{-1} \in U_{t} \Omega_{\tau}^{-1}
$$

be given. In order to obtain $U_{b}$ and $\Omega_{\alpha}$ satisfying (14), we write

$$
\alpha^{-1} b=\alpha(\alpha \alpha)^{-1} \cdot(b \beta) \beta^{-1} \in U_{t} \Omega_{\tau}^{-1} .
$$

Since multiplication is assumed to be continuous in $(T, \cdot, \mathfrak{T} \#(\subseteq \times \mathfrak{G}))$, there are $U_{\alpha}, \Omega_{\alpha \alpha}, U_{b \beta}$ and $\Omega_{\beta}$ such that

$$
U_{\alpha}\left(\Omega_{\alpha \alpha}\right)^{-1} \cdot U_{b \beta} \Omega_{\beta}^{-1} \subseteq U_{t} \Omega_{\tau}^{-1} .
$$

From this follows that $U_{b}$ and $\Omega_{\alpha}$ with $U_{b} \beta \subseteq U_{b \beta}$ and $\Omega_{\alpha} \alpha \subseteq \Omega_{\alpha \alpha}$ satisfy (14).

THEOREM 8. Let $S=\left(S, \cdot, S_{)}\right.$and $T=Q_{r}(S, \Sigma)$ be given and let $G$ be a subgroup of $T$ such that $G \cap S=\Sigma$. Assume further that $(\Sigma, \cdot, \mathfrak{s})$ is a topological semigroup for a topology $\mathfrak{s}$ satisfying (i)-(v) and (14), by which $\left(T, \cdot, \mathfrak{T}^{\#}(\mathfrak{S} \times \mathfrak{s})\right)$ is a topological semigroup. If $\mathfrak{\mathfrak { Z }}=\subseteq \mid \Sigma$, then $(G, \cdot, \mathfrak{T} \#(\mathfrak{S} \times \mathfrak{Z}) \mid G)$ is a topological group.

Proof. A base for $\mathfrak{T}^{\#}(\mathfrak{S} \times \mathfrak{B}) \mid G$ is given by all intersections

$$
U \Omega^{-1} \cap G, \quad U \in \mathfrak{S}, \Omega \in \mathfrak{Z} \text {. }
$$

For each $a \alpha^{-1} \in U \Omega^{-1} \cap G, a \in U, \alpha \in \Sigma$ it follows $a \in G$, hence $a \in U \cap G$ $=U \cap G \cap S=U \cap \Sigma=\Omega^{\prime} \in \mathfrak{g}$. Therefore

$$
U \Omega^{-1} \cap G=\Omega^{\prime} \Omega^{-1}, \quad \Omega^{\prime}, \Omega \in \mathfrak{g}
$$

is a base for $\mathfrak{T}^{\#}(\mathfrak{S} \times \mathfrak{g}) \mid G$, and so $g \rightarrow g^{-1}$ clearly is continuous.

Of course, this theorem is applicable to each subgroup of $T$, depending on a suitable choice of $\Sigma$. We remark, that Theorem 8 is a generalization of [2, Theorem 4], where the (commutative) case with $\Sigma=S$ is considered. In the same way most of the results in $[2, \S 3]$ are covered by our investigations, for instance [2, Theorem 6] by the next theorem, which gives a sufficient condition for $\mathfrak{T} \#(\mathfrak{S} \times \mathfrak{g}) \mid S=\mathfrak{S}$.

THEOREM 9. Let $(S, \cdot, \mathfrak{S})$ and $T=Q_{r}(S, \Sigma)$ be given and suppose that $(\Sigma, \cdot, \mathfrak{s})$ is a topological semigroup for a topology $\mathfrak{s}$ which obeys (i)-(v) and (14). Hence

$$
\left(T, \cdot, \mathfrak{T}^{\#}(\mathfrak{S} \times \mathfrak{s})\right)
$$

is a topological semigroup and $\mathfrak{I}^{\#}(\mathfrak{S} \times \mathfrak{S}) \mid S \subseteq \subseteq$ holds. If there is a topology $\mathfrak{T}_{0}$ on $T$, such that $\left(T, \cdot, \mathfrak{I}_{0}\right)$ is a topological semigroup, $\mathfrak{I}_{0} \mid S=\mathfrak{S}$, and $\mathfrak{\Xi}_{0} \subseteq \mathfrak{g}$ for the topology $\mathfrak{I}_{0}$ corresponding to $\mathfrak{I}_{0}$ by $(12)$, then it follows $\mathfrak{T}^{\#}(\mathfrak{S} \times \mathfrak{I}) \mid S=\mathfrak{S}$.

Proof. From Theorem 5 and $\mathfrak{s}_{0} \subseteq \mathfrak{g}$ we obtain

$$
\mathfrak{I}_{0} \subseteq \mathfrak{T}^{\#}\left(\mathfrak{S} \times \mathfrak{I}_{0}\right) \subseteq \mathfrak{T}^{\#}(\mathfrak{S} \times \mathfrak{\mathfrak { B }}),
$$

which implies

$$
\mathfrak{S}=\mathfrak{I}_{0}|S \subseteq \mathfrak{T} \#(\mathfrak{S} \times \mathfrak{S})| S \subseteq \mathfrak{S} .
$$


Note that $\mathfrak{Z}_{0} \subseteq \mathfrak{S}$ holds if $\left(G, \cdot, \mathfrak{I}_{0} \mid G\right)$ is a topological group and $\subseteq \mid \Sigma \subseteq \mathfrak{Z}$, hence especially if $\Sigma=S, \mathfrak{b}_{0}=\mathfrak{s}=\mathfrak{S}$.

Finally we state without proof the following generalization of Theorem 2.

THEOREM 10. If $(S, \cdot, \subseteq), T=Q_{r}(S, \Sigma)$ and $(\Sigma, \cdot, \mathfrak{b})$ satisfy the assumptions of Theorem 9 and if $(S, \cdot, \subseteq)$ and $(\Sigma, \cdot, \mathfrak{s})$ both are locally compact Hausdorff spaces, then the same is true for $\left(T, \cdot, \mathfrak{T}^{\#}(\mathfrak{S} \times \mathfrak{s})\right)$.

REMARK. The statements of this paragraph are valid with obvious modifications when one considers again semitopological semigroups instead of topological semigroups. We only mention that concerning Theorem 7 one needs no further assumption to prove the continuity of the multiplication from the right side, whereas for the other side (14) is to be replaced by $\Omega_{\alpha}^{-1} b \subseteq t \Omega_{\tau}^{-1}$.

7. Semigroups satisfying the condition (5). Let $S=(S, \cdot, \subseteq)$ be a topological semigroup, let $T=Q_{r}(S, \Sigma)$ be a semigroup of right quotients of $S$, and assume that (5) is fulfilled. We are going to apply the preceding results to this case for some topologies 3 on $\Sigma$.

First we consider the discrete topology $\mathfrak{b}_{d}$ on $\Sigma$. Obviously property (v) of Theorem 6 is satisfied, because $x \xi^{-1} \in U \alpha^{-1}, U \in \subseteq$ implies $x l \subseteq U \lambda$ for $\xi l=\alpha \lambda$, and by (5) we have $U_{x} l \subseteq U \lambda$ for some $U_{x} \in \mathfrak{S}$, hence $U_{x} \xi^{-1} \subseteq U \alpha^{-1}$. Therefore

$$
\mathfrak{B}\left(\mathfrak{S}_{,} \mathfrak{S}_{d}\right)=\left\{U \alpha^{-1} \mid U \in \mathbb{S}, \alpha \in \Sigma\right\}
$$

is a base for the topology $\mathfrak{T}^{\sharp}\left(\mathfrak{S} \times \mathfrak{g}_{d}\right)$ on $T$, just the topology considered in Theorem 1. Moreover, condition (14) reduces to

$$
\alpha^{-1} b \in U_{t} \tau^{-1} \Rightarrow \alpha^{-1} U_{b} \subseteq U_{t} \tau^{-1} \quad \text { for some } U_{b},
$$

and the existence of such a $U_{b}$ is an immediate consequence of (5). Hence Theorem 7 again proves $\left(T, \cdot, \mathfrak{T}^{\#}\left(\mathfrak{S} \times \mathfrak{S}_{d}\right)\right)$ to be a topological semigroup. From our considerations in $\S 3-\S 5$ we know $\mathfrak{T}^{\sharp}\left(\mathfrak{S} \times \mathfrak{\mathfrak { B }}_{d}\right) \mid S=\mathfrak{S}$ and that each subgroup $G$ of $T$ is a topological group with respect to $\mathfrak{T}^{\#}\left(\mathfrak{S} \times \mathfrak{S}_{d}\right) \mid G$ if and only if $F_{r}\left(\Sigma, \Sigma^{\prime}\right)$ holds; there are examples where $\left(G, \cdot, \mathfrak{I}^{\sharp}\left(\mathfrak{S} \times \mathfrak{I}_{d}\right) \mid G\right)$ merely is a topological semigroup, but not a topological group.

Next we shall regard different subsemigroups $\Sigma_{1}, \Sigma_{2}, \ldots$ corresponding to the same semigroup $T=Q_{r}\left(S, \Sigma_{1}\right)=Q_{r}\left(S, \Sigma_{2}\right)=\cdots$ and we take all such $\Sigma_{i}$ for which there exists a subgroup $G_{i}$ of $T$ such that $\Sigma_{i}=G_{i} \cap S$. Now we consider the topologies $\mathfrak{\xi}_{i}=\mathfrak{S} \mid \Sigma_{i}$ on $\Sigma_{i}$. By Corollary 1, $\S 3$, the condition (5) is fulfilled for all $\Sigma_{i}$ simultaneously.

THEOREM 11. Let $S=\left(S, \cdot, S_{)}\right.$be a topological semigroup, let $T=Q_{r}\left(S, \Sigma_{i}\right)$ be a semigroup of right quotients, containing a subgroup $G_{i}$ with $G_{i} \cap S=\Sigma_{i}$, and assume (5). Then the conditions (i)-(v) of Theorem 6 hold for $\mathfrak{\xi}_{i}=\varsigma \mid \Sigma_{i}$.

Proof. We show (v), using the remark behind Theorem 6. Let $U_{a} \in \mathbb{S}$ and $\Omega_{\alpha} \in \check{\zeta}_{i}$ be given and take $U_{\alpha} \in \mathbb{S}$ with $U_{\alpha} \cap \Sigma_{i}=\Omega_{\alpha}$. Since $x l=a \lambda \in U_{a} \lambda \in \mathbb{S}$ and 
$\xi l=\alpha \lambda \in U_{\alpha} \lambda \in \mathbb{S}$, there are $U_{x}, U_{\xi} \in \mathfrak{S}$ such that $U_{x} l \subseteq U_{a} \lambda, U_{\xi} l \subseteq U_{\alpha} \lambda$. For each $x^{\prime} \in U_{x}, \xi^{\prime} \in U_{\xi} \cap \Sigma_{i}=\Omega_{\xi}$ we have $x^{\prime} l=a^{\prime} \lambda, \xi^{\prime} l=u \lambda$ for some $a^{\prime} \in U_{a}, u \in U_{\alpha}$. From $l=\xi^{-1} \alpha \lambda \in G_{i}$ it follows $u=\xi^{\prime} l \lambda^{-1} \in G_{i}$, hence $u \in G_{i} \cap S=\Sigma_{i}$ and $u \in U_{\alpha} \cap \Sigma_{i}=\Omega_{\alpha}$ as we were to show.

So for each $\mathfrak{\mathfrak { B }}_{i}=\mathfrak{\subseteq} \mid \Sigma_{i}$ as above

$$
\mathfrak{B}\left(\mathfrak{S}, \mathfrak{S}_{i}\right)=\left\{U \Omega^{-1} \mid U \in \mathfrak{S}, \Omega \in \mathfrak{B}_{i}\right\}
$$

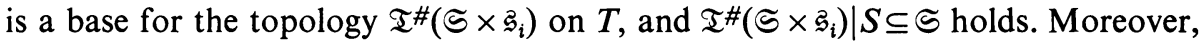
$\left(T, \cdot, \mathfrak{T} \#\left(\widetilde{S} \times \mathfrak{s}_{i}\right)\right)$ is a topological semigroup if and only if property (14) of Theorem 7 is satisfied. If this is the case (especially, if $\Sigma_{i}$ is contained in the centre of $S$ ), then $\left(G_{i}, \cdot, \mathfrak{T}^{\#}\left(\subseteq \times \mathfrak{S}_{i}\right) \mid G_{i}\right)$ is a topological group by Theorem 8 . As a special case we obtain the theorem due to [2] mentioned in the introduction.

We further remark that from $G_{1} \supseteq G_{2}$, i.e., $\Sigma_{1} \supseteq \Sigma_{2}$, it follows

$$
\mathfrak{T}^{\#}\left(\mathfrak{S} \times \mathfrak{s}_{1}\right) \subseteq \mathfrak{T}^{\#}\left(\mathfrak{S} \times \mathfrak{s}_{2}\right) \subseteq \mathfrak{T}^{\#}\left(\mathfrak{S} \times \mathfrak{s}_{d}\right),
$$

where the topology $\mathfrak{I}^{\sharp}\left(\subseteq \times \mathfrak{S}_{d}\right)$ does not depend on the choice of $\Sigma_{i}$ for

$$
T=Q_{r}\left(S, \Sigma_{1}\right)=Q_{r}\left(S, \Sigma_{2}\right) .
$$

Finally, we take the condition $F_{r}\left(\Sigma_{i}, \Sigma_{i}\right)$ (or, equivalently, $F_{l}\left(\Sigma_{i}, \Sigma_{i}\right)$ ) into consideration. One can prove that $F_{r}\left(\Sigma_{i}, \Sigma_{i}\right)$ and (5) imply (14) for $\mathfrak{T}^{\sharp}\left(\subseteq \times \mathfrak{s}_{i}\right)$, but we obtain a stronger result as follows:

THEOREM 12. Under the same assumptions as in Theorem 11, the topology $\mathfrak{I} \#\left(\mathfrak{S} \times \mathfrak{S}_{i}\right), \mathfrak{S}_{i}=\mathfrak{S} \mid \Sigma_{i}$, corresponding to (16) coincides with the topology $\mathfrak{I} \sharp\left(\subseteq \mathfrak{S}_{d}\right)$ corresponding to (15), if and only if $F_{r}\left(\Sigma_{i}, \Sigma_{i}\right)$ holds.

Proof. Applying Theorem 5 to the topology $\mathfrak{I}=\mathfrak{T} \#\left(\subseteq \times \mathfrak{g}_{d}\right)$ on $T=Q_{r}\left(S, \Sigma_{i}\right)$, there is a topology

$$
\mathfrak{T}^{\#}(\mathfrak{S} \times \mathfrak{s}) \supseteq \mathfrak{T}=\mathfrak{T}^{\#}\left(\mathfrak{S} \times \mathfrak{I}_{d}\right)
$$

on $T$ for a topology $\mathfrak{s}$ on $\Sigma_{i}$ defined by (12). Now suppose $F_{r}\left(\Sigma_{i}, \Sigma_{i}\right)$. Then

$$
\left(G_{i}, \cdot, \mathfrak{T} \#\left(\mathfrak{S} \times \mathfrak{b}_{d}\right) \mid G_{i}\right)
$$

is a topological group by Theorem 3. Hence the Supplement of Theorem 5 is applicable, and the topology $\mathfrak{s}$ from (12) coincides with

$$
\mathfrak{I}\left|\Sigma_{i}=\mathfrak{T} \#\left(\mathfrak{S} \times \mathfrak{b}_{d}\right)\right| \Sigma_{i}=\mathfrak{I} \#\left(\mathfrak{S} \times \mathfrak{b}_{d}\right)|S| \Sigma_{i}=\mathfrak{S} \mid \Sigma_{i}=\mathfrak{S}_{i} .
$$

From $\mathfrak{B}=\mathfrak{s}_{i}$ and (18) we obtain

$$
\mathfrak{I}^{\#}\left(\mathfrak{S} \times \mathfrak{I}_{i}\right) \supseteq \mathfrak{I}^{\#}\left(\mathfrak{S} \times \mathfrak{a}_{d}\right),
$$

and from this and (17) we have $\mathfrak{T} \#\left(\subseteq \times \mathfrak{S}_{i}\right)=\mathfrak{T} \#\left(\subseteq \times \mathfrak{S}_{d}\right)$.

Conversely, using this equality, $\left(T, \cdot, \mathfrak{I} \#\left(\subseteq \times \mathfrak{S}_{\mathfrak{i}}\right)\right)$ is a topological semigroup, hence we can apply Theorem 8 , from which $\left(G_{i}, \cdot, \mathfrak{T}^{\#}\left(\mathfrak{S} \times \mathfrak{J}_{d}\right) \mid G_{i}\right)$ is a topological group, and $F_{r}\left(\Sigma_{i}, \Sigma_{i}\right)$ follows from Theorem 3. 
Of course, looking at (17), from Theorem 12 follows the same statement for each topology $\mathfrak{I}^{\#}\left(\mathfrak{S} \times \mathfrak{S}_{j}\right)$ corresponding to a $\Sigma_{j} \subset \Sigma_{i}$, whereas for $\Sigma_{j} \supset \Sigma_{i}$ the topology $\mathfrak{I}^{\#}\left(\mathfrak{S} \times \mathfrak{S}_{j}\right)$ may differ from $\mathfrak{T} \#\left(\mathfrak{S} \times \mathfrak{I}_{i}\right)=\mathfrak{T}^{\#}\left(\mathfrak{S} \times \mathfrak{S}_{d}\right)$.

\title{
REFERENCES
}

1. A. H. Clifford and G. B. Preston, The algebraic theory of semigroups. Vols. I, II, Math. Surveys, no. 7, Amer. Math. Soc., Providence, R. I., 1961, 1967. MR 24 \#A2627; MR 36 \#1558.

2. B. Gelbaum, G. K. Kalisch and J. M. H. Olmsted, On the embedding of topological semigroups and integral domains, Proc. Amer. Math. Soc. 2 (1951), 807-821. MR 13, 206.

3. T. Husain, Introduction to topological groups, Saunders, Philadelphia, Pa., 1966. MR 34 \#278.

4. K. Keimel, Lokal kompakte Kegelhalbgruppen und deren Einbettung in topologische Vektorräume, Math. Z. 99 (1967), 405-428.

5. J. L. Kelley, General topology, Van Nostrand, Princeton, N. J., 1955. MR 16, 1136.

6. A. B. Paalman de Miranda, Topological semigroups, Mathematisch Centrum, Amsterdam, 1964. MR 29 \#5228.

7. N. J. Rothman, Embedding of topological semigroups, Math. Ann. 139 (1960), 197-203. MR 22 \#6871.

8. E. Schieferdecker, Einbettungssätze für topologische Halbgruppen, Math. Ann. 131 (1956), 372-384. MR 18, 658.

9. D. Tamari, Sur l'immersion d'un semi-groupe topologique dans un groupe topologique, Algèbre et Théorie des Nombres, Centre National Recherche Scientifiques, Paris, 1950, pp. 217-221. MR 12, 670.

10. H. J. Weinert, Über Halbringe und Halbkörper. II, Acta Math. Acad. Sci. Hungar. 14 (1963), 209-227. MR 26 \#6219.

11. - Zur Erweiterung algebraischer Strukturen durch Rechtsquotientenbildung, Acta Math. Acad. Sci. Hungar. 16 (1965), 213-214. MR 30 \#3932.

12. - On the extension of partial orders on semigroups of right quotients, Trans. Amer. Math. Soc. 142 (1969), 345-353.

\author{
UNIVERSITY OF FLORIDA, \\ GainesVille, Florida \\ JOHANNES GUTENBERG UNIVERSITÄT, \\ MaINZ, GeRMANY
}

